\title{
Surgical Management of Lower Extremity Lymphedema: A Comprehensive Review
}

\author{
Pedro Ciudad ${ }^{1,2}$ M. Diya Sabbagh ${ }^{3}$ Mouchammed Agko ${ }^{4}$ Tony C.T. Huang ${ }^{3}$ Oscar J. Manrique ${ }^{3}$ \\ Carmen Román L. ${ }^{5}$ Cesar Reynaga ${ }^{1}$ Ricardo Delgado ${ }^{6}$ Michele Maruccia ${ }^{1}$ Hung-Chi Chen ${ }^{2}$
}

1Department of Plastic, Reconstructive and Burn Surgery, Arzobispo
Loayza National Hospital, Lima, Peru
2Department of Plastic and Reconstructive Surgery, China Medical
University Hospital, Taichung, Taiwan
${ }^{3}$ Division of Plastic and Reconstructive Surgery, Mayo Clinic,
Rochester, Minnesota, United Sates
${ }^{4}$ Section of Plastic Surgery, Medical College of Georgia, Augusta
University, Augusta, Georgia
${ }^{5}$ Department of Physical and Rehabilitation Medicine, National
Cancer Institute of Peru (INEN), Lima, Peru
6 Department of Plastic and Reconstructive Surgery, National Cancer
Institute of Peru (INEN), Lima, Peru
Indian J Plast Surg 2019;52:81-92

Address for correspondence Pedro Ciudad, MD, PhD, Department of Plastic, Reconstructive and Burn Surgery, Arzobispo Loayza National Hospital, Avenida Alfonso Ugarte 848, Cercado de Lima 15082, Peru (e-mail: pciudad@hotmail.com).
Abstract
Keywords
- lymphedema
- vascularized lymph node transfer
- radical reduction in lymphedema with preservation of perforators
- suction-assisted lipectomy
- lower extremity lymphedema

Lymphedema refers to the accumulation of protein-rich fluid in the interstitial spaces. This can occur secondary to congenital malformation of the lymphatic channels or nodes or as a result of an insult that damages appropriately formed channels and nodes. Stagnant, protein-rich lymph initiates an inflammatory response that leads to adipocyte proliferation, fibrous tissue deposition, and increased susceptibility to infections. The end result is permanent disfigurement and dermal changes. Early and accurate diagnosis is essential, since lymphedema is a chronic and progressive problem. When lymphedema affects the lower extremity, it is important to manage it in a way that preserves function and mobility. Early diagnosis also allows for a proactive rather than reactive approach to treatment and utilization of novel physiologic procedures, such as lymphovenous anastomosis and vascularized lymph node transfer. Such interventions slow down disease progression and reduce morbidity by allowing the surgeon to salvage the remaining functional lymphatic channels. When physiologic procedures fail or when faced with a delayed presentation, the addition of excisional procedures can provide a more comprehensive treatment of this debilitating disease. The aim of this article is to review the most current concepts in the surgical management of lower extremity lymphedema.

\section{Introduction}

The term lymphedema is used to describe a chronic, progressive clinical condition characterized by edema that arises secondary to accumulation of protein-rich lymphatic fluid in the interstitial space. ${ }^{1,2}$ The main function of the lymphatic system is to remove the excess interstitial fluid from different regions of the body and return it to the blood circulation. ${ }^{3,4}$ The lymphatic system also plays an important role in regulating immune response, transportation of immune cells, in addition to self and foreign antigen processing. ${ }^{5}$ Primary lymphedema arises when the lymphatic channels or nodes are structurally or functionally impaired or underdeveloped. This can be due to obstructed, malformed, or hypoplastic lymphatic pathways. The lower extremities are more likely to be involved in cases of primary lymphedema. The exact cause remains unknown; however, gravity probably exacerbates the problem and contributes to its progression..$^{6-9}$
DOI https://doi.org/

10.1055/s-0039-1688537

ISSN 0970-0358.
(C)2019 Association of Plastic Surgeons of India
License terms

$($ (1) $\odot \circledast$ 
Secondary lymphedema refers to lymphedema caused by obstruction or destruction of normally formed and developed lymphatic channels or nodes. This can occur following trauma, radiation, infection, or surgery. In the developing countries, the most common cause is parasitic infection, mostly due to Wuchereria bancrofti. ${ }^{10}$

It is estimated that up to 300 million people around the world suffer from lymphedema. While the majority of these cases in developing countries are secondary to the aforementioned parasitic filarial disease, in developed countries most cases occur secondary to malignancy or malignancy-related treatment. ${ }^{11-13}$ Compared with breast cancer-related upper extremity lymphedema, the incidence of postoncologic treatment lower extremity lymphedema (LEL) is noted to be higher with gynecologic cancers. ${ }^{14}$ Reports vary widely due to the diverse underlying pathology, oncologic treatment protocol, and lymphedema assessment method. Nevertheless, the following ranges have been documented in the literature: 1.2 to $47 \%$ from endometrial cancer, 0 to $55.9 \%$ from cervical cancer, and 4.7 to $40.8 \%$ from ovarian cancer. ${ }^{15}$ The incidence of LEL is similarly high in patients undergoing inguinal lymph node dissection for melanoma and can reach up to $55 \% .{ }^{16}$ In contrast, there is a dearth of publications on the prevalence of lymphedema in urological cancers. Even though lymphedema can develop in 50 to $100 \%$ of patients with penile carcinoma with nodal metastasis undergoing inguinal dissection, it is less common in other urological cancers. ${ }^{17}$ Rates from prostate cancer range from 0 to $38 \%$ depending on the extent of lymphadenectomy and adjuvant radiation..$^{18}$ The majority of patients present with LEL within the first year after the initial insult and the disease tends to progress faster than upper extremity lymphedema. ${ }^{19}$

Regardless of the underlying etiology, lymph accumulation and stasis in the interstitial space trigger an inflammatory response that leads to adipose tissue proliferation and fibrous tissue deposition. The result is mild-to-severe permanent edema of the affected body part. Edema can be further complicated by infections and wound healing problems resulting in induration and disfigurement that can cause pain, discomfort, and compromised mobility. The problem is progressive, as the accumulation of lymphatic fluids and associated inflammation leads to more destruction of the remaining functional lymphatic channels. ${ }^{4,20}$ Lymphedema results in a significant pain, morbidity, and affects the quality of life negatively. It also results in significant financial burden on both the patient and the healthcare system..$^{21-23}$

To this date, there is no definite cure for lymphedema. Lymphedema has been traditionally managed conservatively with complete decongestive therapy that encompasses manual lymphatic drainage, compression therapy, and skin/wound care. Surgical treatment was only attempted when lymphedema became refractory to conservative measures and was often complicated with recurrent infections and chronic wounds. The surgical approach was mainly excisional and aimed to remove to differing degrees the diseased skin and subcutaneous tissue. Such procedures, however, were associated with significant blood loss, morbidity, infections, as well as permanent disfigurement and recurrence of symptoms $\mathrm{s}^{24,25}$ ( - Fig. 1).
In the recent years, advances in microsurgery and improved understanding of the lymphatic system and pathogenesis of lymphedema have allowed the introduction of novel physiologic procedures such as lymphovenous anastomosis (LVA) and vascularized lymph node transfer (VLNT). These procedures have shown significant promise in the management of lymphedema, both in animal and clinical studies. ${ }^{26-28}$ In addition, modification of procedures such as Charles', introduction of innovative approaches such as radical reduction of lymphedema with preservation of perforators and application of suction-assisted lipectomy (SAL) has open new horizons in the surgical treatment of LEL. By strategically combining physiologic and excisional procedures, safer and more predictable outcomes can be achieved.

This article reviews the contemporary surgical management of LEL with emphasis on accurate diagnosis and algorithmic approach to selection of the appropriate treatment protocol.

\section{Diagnosing Lower Extremity Lymphedema}

A common presenting symptom in lymphedema patients is swelling of the affected limb. Thus, confirmation of lymphedema as the cause of this is of primary importance. The next step would be objective quantification of change in the limb size. Several criteria have been used to diagnose lymphedema: difference in limb circumferences more than $2 \mathrm{~cm}$, limb volume differences more than $200 \mathrm{ml}$, or at least $5 \%$ limb volume change. ${ }^{29-32}$ However, all these measures merely confirm edema but do not confirm the underlying etiology. Maclellan et al reported that $25 \%$ of patients referred to a lymphedema specialist did not have true lymphedema. ${ }^{33}$ On the other hand, symptoms of heaviness and discomfort can be reported by the patients well before any edema becomes clinically evident. Thus, a thorough history and physical examination is still crucial in identifying the etiology, precipitating factors, disease course, relevant family history, and previous treatments. Both the involved and healthy appearing extremities should be examined to determine extent and stage of the disease. Decreased skin pliability results in a positive "Stemmer sign." This refers to the inability of the examiner use the thumb and index finger to pinch dorsal skin of a toe.,34 Further diagnostic imaging is then undertaken to evaluate lymphatic function and, if indicated, exclude nonlymphogenic edema (i.e., venous disease).

Objectively, several diagnostic models can be used to confirm the presence of lymphedema and follow disease progression. Radionuclide lymphoscintigraphy is one such modality that is available in many centers where the transport index of a radio nucleotide from the injection site to the lymph nodes basin can be calculated to quantify the severity of the disease. Lymphoscintigraphy can also be used to visualize the lymphatic channels and evaluate for any structural abnormalities or obstruction. . $35,36^{\text {Magnetic resonance }}$ lymphography (MRL) is more expensive but provides superior insight into the status of the lymphatic system and the soft tissue changes associated with lymphedema. Lastly, indocyanine green (ICG) lymphangiography can be used with near-infrared 


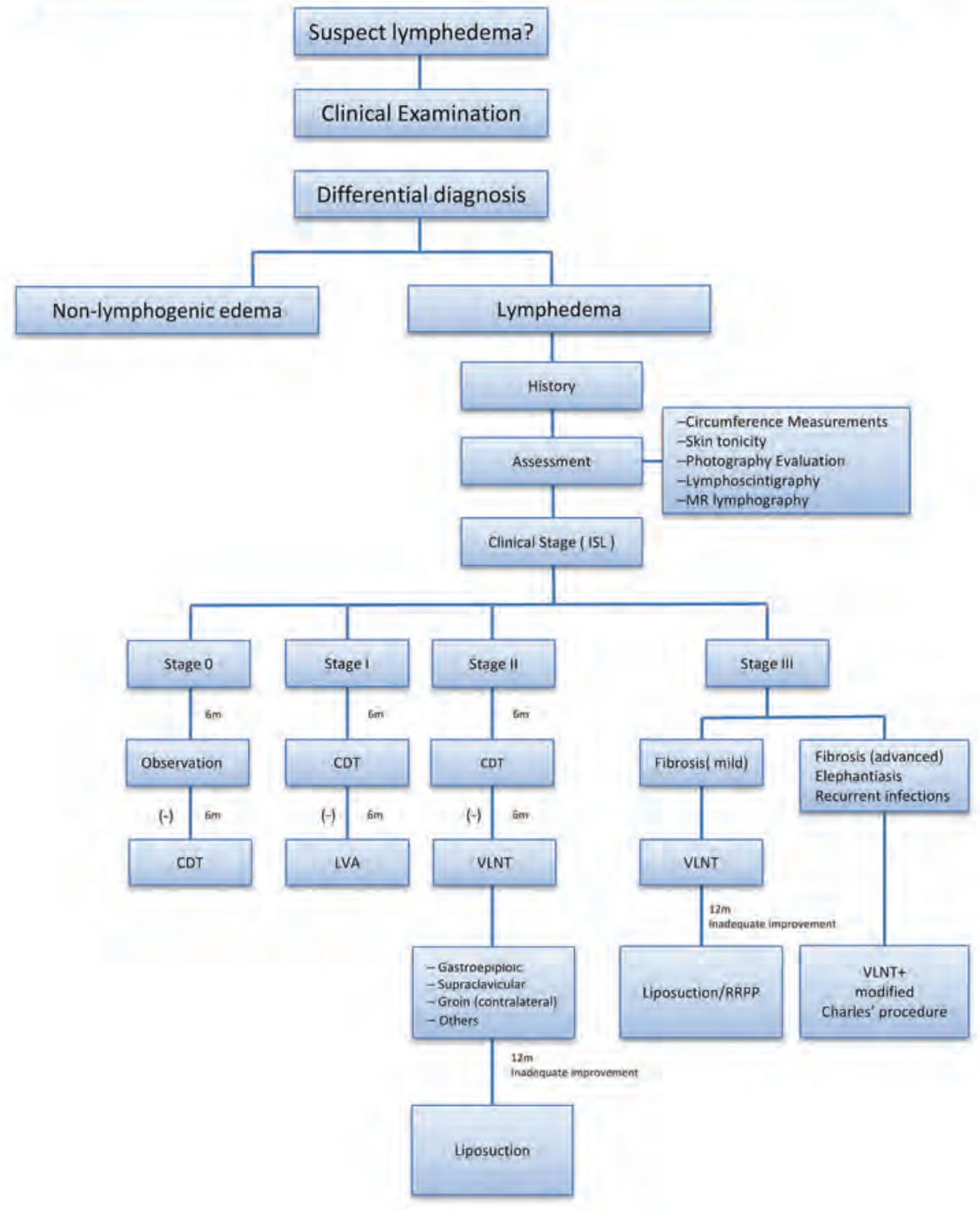

Fig. 1 An algorithmic approach is essential for the clinical management of extremity lymphedema. To achieve better outcomes, it helps to optimize the use of diagnostic modalities. For lower extremity lymphedema, the management rests on eliciting detailed history and performing thorough clinical examination. This is effectively complemented by other diagnostic modalities. The options range from simple circumference measurements, measurement of skin tonicity to imaging studies like lymphoscintigraphy and magnetic resonance lymphangiograms. Photographic documentation also plays an important role. The entire evaluation process helps to ascertain whether the etiology is systemic or local, congenital or acquired. Correct diagnosis is the cornerstone in selecting the right procedure for the right patient. The stage of severity can then be decided as per the International Society of Lymphology staging criteria. Stage 0 is the preclinical stage. For these patients, we suggest regular observation for up to 6 months. Depending on whether the swelling subsides or increases, they may be further observed or subjected to compression decongestive therapy (CDT). Stage 1 patients are immediately started on CDT after diagnosis. The decision to intervene is taken 6 months later. The patients who do not respond satisfactorily are ideal candidates for lymphovenous anastomosis (LVA). For stage 2 patients, 6 -month trial of CDT may be tried first. The patients with persistent or increasing symptoms vascularized lymph node transfer (VLNT) are offered. The commonly performed vascularized lymph node flaps are gastroepiploic, supraclavicular, and groin. These patients are offered liposuction, if the reduction is not satisfactory at the end of 12 months. For stage 3 patients, CDT trial is not mandatory and immediate intervention is considered. If mild fibrosis is seen, VLNT is performed. Twelve months later (depending on the improvement), one can plan debulking procedure in the form of liposuction or radical reduction in lymphedema with preservation of perforators. However, if the fibrosis is severe along with recurrent infection and features of elephantiasis, debulking with modified Charles' procedure is the procedure of choice. Ciudad P, Agko M, Chen HC. LYMPHEDEMA - Surgical Approach and Specific Topics. Elsevier Taiwan, Algorithm for the Surgical Management of Lymphedema, 188-189.

cameras that visualize the lymphatic system in real time. After injection of ICG distally (web spaces), this modality enables the visualization of the lymphatic channels and nodes. It also allows the identification of patent lymphatic channels that can be used to treat lymphedema surgically. The advantages of this technique are that it is quick, safe, can be used in patients who 
cannot undergo MRL due to metal implants or contrast allergy. It can also be done immediately prior to the surgery for site marking and lymphatic channels identification. ${ }^{26,37,38}$

Despite the fact that several clinically more relevant classifications have been proposed in literature, the most widely accepted across disciplines is the International Society of Lymphology (ISL) (-Table 1). ${ }^{2}$ In ISL Stage 0 lymphedema, there is demonstrable lymphatic dysfunction with ICG lymphangiography with abnormal dermal backflow patterns. While patients may complain of subjective symptoms, edema is not clinically evident. As lymphatic function deteriorates, edema becomes evident and progesses usually in a distal to proximal fashion (ISL Stage I). On physical examination, pitting edema can be appreciated. However, at this stage edema is reversible with elevation. The transition to irreversible edema denotes ISL Stage II. Pitting, which is common in early Stage II, becomes less detectable in late Stage II as subcutaneous adipose tissue proliferation and fibrosis ensues. Finally, lymphostatic elephantiasis (ISL Stage III) is characterized by progressive edema, absence of pitting edema, visible disfigurement, and substantial skin changes such as hyperkeratosis and polypoid nodules.

\section{Surgical Treatment of Lower Extremity Lymphedema}

Once the diagnosis of LEL has been established and the disease severity staged, the patients are best served by a multidisciplinary healthcare team that combines their efforts to offer an optimum treatment. The treatment goals in LEL patient are to improve the functional status, reduce patient's dependence on compression devices, and to decreasethe risk of recurrent infections. Furthermore, any surgical intervention should aim at allowing the patient to be able to utilize footwear.

Conservative management in the form of complete decongestive therapy is usually the first treatment the patients receive and it continues to be an integral part of any surgical treatment protocol both preoperatively and to varied degrees postoperatively ( - Fig. 2 ). In spite of the recent surging interest in microsurgical procedures for lymphedema, there is not a universally accepted algorithm to guide decision making with regard to timing, selection, and possible combinations of the available procedures. Surgeons have reported variable results with a multitude of surgical techniques. As lymphedema progresses through the aforementioned ISL stages, the clinical deterioration reflects the histological changes that take place in the lymphedematous limb. Initially, lymphatic dysfunction leads to fluids stasis in the interstitial place and this induces inflammation. Inflammation in turn causes adipose tissue hypertrophy and progressive fibrosis. Thus, while at early stages lymphatic fluid stagnation is the main reason for the edema, later the swelling becomes irreversible due to the deposition of solid components such as fat and fibrous tissue. It is imperative that the surgical approach planned for any given stage addresses these pathophysiologic changes. Physiologic procedures promote clearance of lymphatic fluid from the interstitial space. However, since they do not directly eliminate the solid component, any surgical plan without an excisional component would fall short of fully addressing the issue at hand. This is particularly true for the more advanced stages.

\section{Physiologic Procedures}

As mentioned earlier, physiologic techniques improve lymphatic drainage through redirecting lymphatic drainage into the venous system or by inducing the formation of new lymphatic channels through lymphangiogenesis. Among those procedures, LVA and VLNT are among the most popular options to improve lymphatic drainage and have both shown encouraging results clinically.

\section{Lymphovenous Anastomosis}

LVA is a procedure where the excess lymph is directed into the venous circulation. Superficial lymphatic vessels are identified preoperatively using ICG. The procedure entails injecting 0.5 to $1.0 \mathrm{~mL}$ of ICG subcutaneously distally in the limb such as the web space. Near-infrared cameras are then used to identify functioning lymphatic channels. Those channels are marked and incisions are made over the areas that they are in a vicinity of a superficial vein. Isoflurane

Table 1 International Society of Lymphology classification of lymphedema

\begin{tabular}{|l|l|}
\hline Stage 0 & $\begin{array}{l}\text { No edema (latent/subclinical) } \\
\text { - Swelling is not yet evident despite impaired lymph transport, subtle changes in tissue fluid/ } \\
\text { composition, and changes in subjective symptoms } \\
\text { - It may exist months or years before overt edema occurs }\end{array}$ \\
\hline Stage I & $\begin{array}{l}\text { Reversible edema (with elevation) } \\
\text { - Fluid relatively high in protein content } \\
\text { - Pitting (+/-) } \\
\text { - Proliferating cells }\end{array}$ \\
\hline Stage II & $\begin{array}{l}\text { Irreversible edema (even with elevation) } \\
\text { - Pitting }(+)\end{array}$ \\
\hline Late & $\begin{array}{l}\text { Excess fat/fibrosis } \\
\text { - Pitting }(+/-)\end{array}$ \\
\hline Stage III & $\begin{array}{l}\text { Lymphostatic elephantiasis } \\
\text { - Pitting }(-)\end{array}$ \\
\hline
\end{tabular}



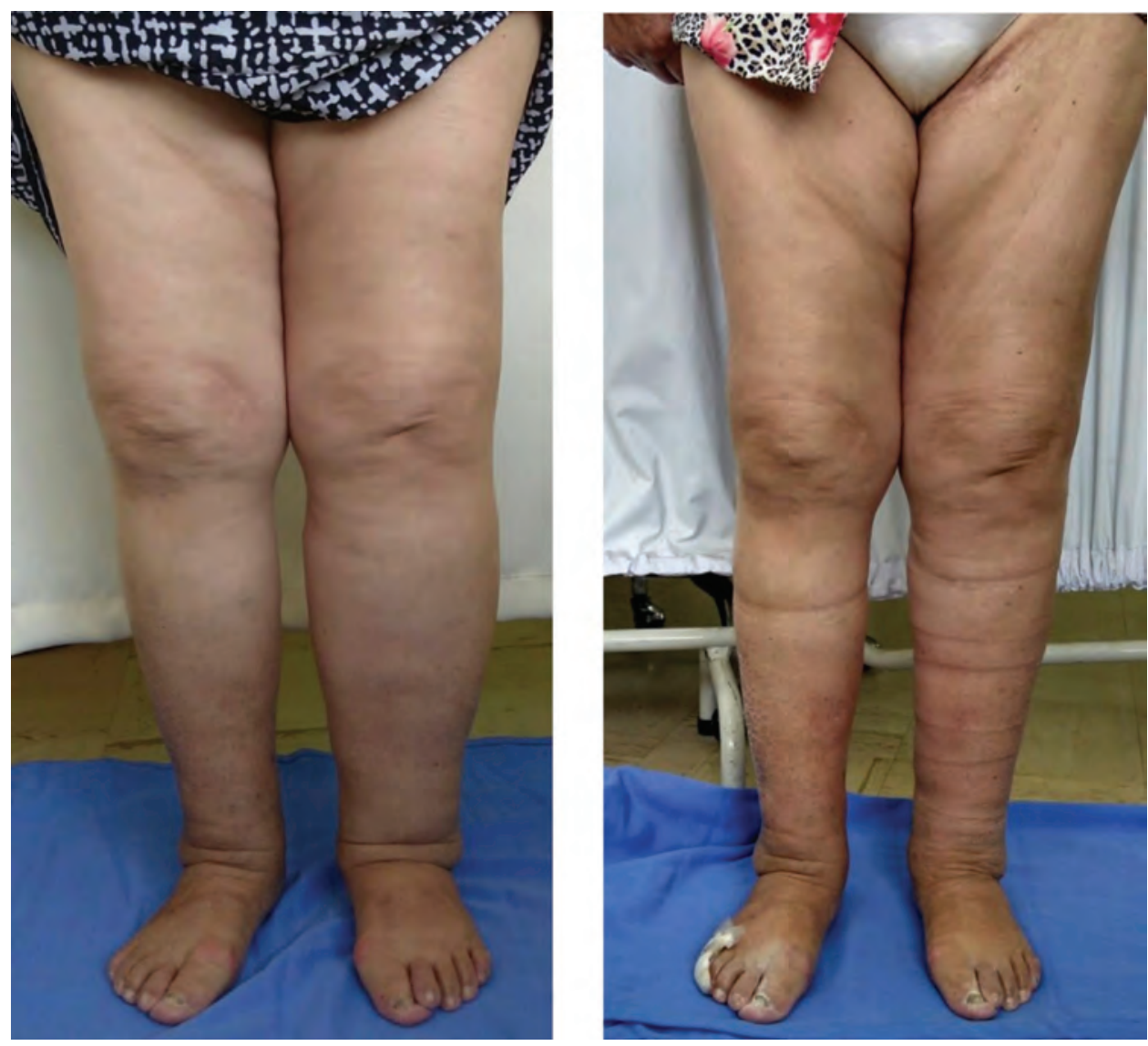

Fig. 2 Patient with left lower extremity lymphedema managed conservatively with complete decongestive therapy (CDT) that encompasses manual lymphatic drainage, compression therapy, and skin/wound care. (A) Pre-CDT. (B) Three months after CDT.

blue dye is also injected subcutaneously just distal to the incision to allow the identification of lymphatic vessels. Once the vessels have been identified, LVA can be done in an end-to-end or end-to-side fashion using super-microsurgical techniques depending on the size discrepancy between the vein and the lymphatic vessels $\mathbf{s}^{4,39}$ ( - Fig. 3). As many LVAs as possible can be done to improve lymph flow.

Multiple studies have demonstrated the efficacy of this technique. ${ }^{40-42}$ In our experience, this procedure is effective in the management of Stage I and early Stage II lymphedema.

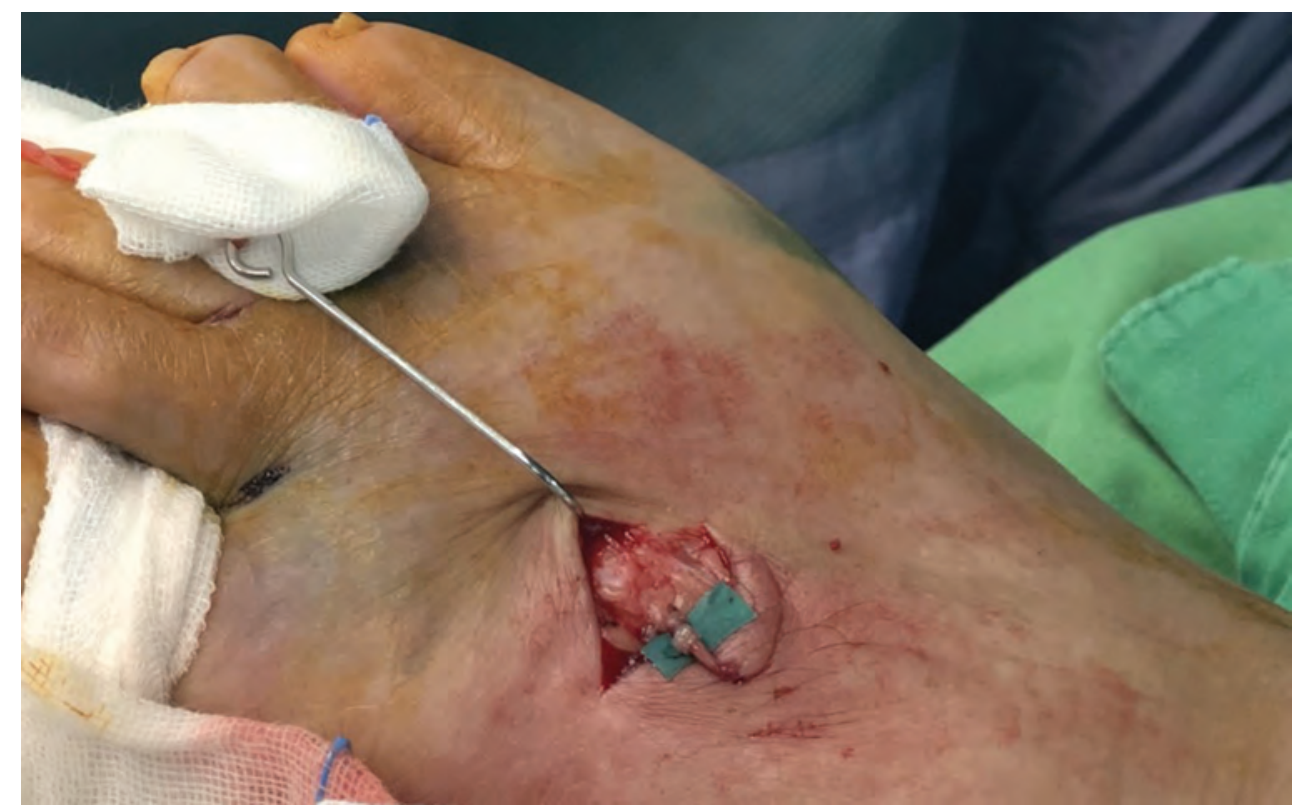

Fig. 3 Anastomotic coupler devices for lymphovenous anastomosis. 
Moreover, possibly due to the dependent position of the lower extremities, LVA is much more effective for upper rather than LEL. ${ }^{43}$ The limb size reduction achieved with LVA can be variable; however, most patients report improvement in symptoms. Generally speaking, patients still need to use compression garments.

\section{Vascularized Lymph Node Transfer}

In patients where functional lymphatic channels or nodes are absent or dysfunctional, such as following lymphadenectomy or radiotherapy, VLNT is an alternative physiologic procedure that improves lymph drainage and flow. The exact mechanism is still unknown and several theories have been proposed. Most likely, the mechanism of action is a combination of those theories. One theory is that the lymph node absorbs the excess lymphatic fluid accumulating in the vicinity. ${ }^{44}$ Another theory is that vascularized lymph nodes induce lymphangiogenesis by releasing vascular endothelial growth factor-C. ${ }^{45,46}$

Various donor sites have been described for vascularized lymph node flap harvest such as the groin, ${ }^{45,47}$ submental, ${ }^{48}$ supraclavicular, ${ }^{49}$ lateral thoracic, ${ }^{50}$ gastroepiploic, ${ }^{51,52}$ jejunal, ${ }^{53}$ appendicular, ${ }^{54}$ and ileocecal ${ }^{55}$ lymph nodes. All of the aforementioned options offer good functional result in terms of management of lymphedema (-Fig. 4). Due to concerns about donor site morbidity and iatrogenic lymphedema in the donor site, our team has explored various options and compared outcomes following VLNTs from different donor sites. ${ }^{56}$ Our preferred donor site is the gastroepiploic lymph node flap for treatment of both upper and LEL. ${ }^{57}$ This is due to its low morbidity, reasonable length of hospital stay, and the fact that it could be harvested laparoscopically (-Figs. 5 and $\mathbf{6}$ ). Furthermore, no cases of instances of iatrogenic lymphedema have been seen by the time of writing this review. It is important to mention, however, that when the gastroepiploic flap is not available due to previous operations, radiation, or for any other reason, we resort to other donor sites. The choice of which is dependent on the surgeon's experience and individualized to each case.

Once the appropriate donor site has been selected and the lymph node flap has been harvested, the choice of inset site is determined based on multiple factors. For instance, severity of lymphedema, scar tissue, and prior radiation as well as the aesthetic appearance are all factors that influence where the lymph node flap will be placed. As for the exact location on the lower limb, several reports have suggested different aspects of the limb: proximal, middle, or distal. Distal placement of the vascularized lymph node where edema is more pronounced appears to be the most effective. This is due to the fact that placing the flap in the most dependent position on the limb may facilitate the pumping and absorption function of the stagnant lymph in addition to the fact that lymph is usually pooled in the most dependent location due to the effect of gravity. Furthermore, distal locations are usually away from radiation site and when insetting the flap in the ankle, there is no need to sacrifice a large artery to use it as the recipient vessel. In the lower extremity, we prefer to use the dorsalis pedis artery as the recipient vessel. This also allows the placement of the flap on the medial aspect of the ankle where it does not affect patient's ability to wear footwear.

Although the aforementioned procedures are effective in controlling diseases progression, it is important to keep in mind that these procedures do not have any reduction effect on the fibrosis and adipocyte hypertrophy that have occurred prior to treatment. This makes such approaches effective in
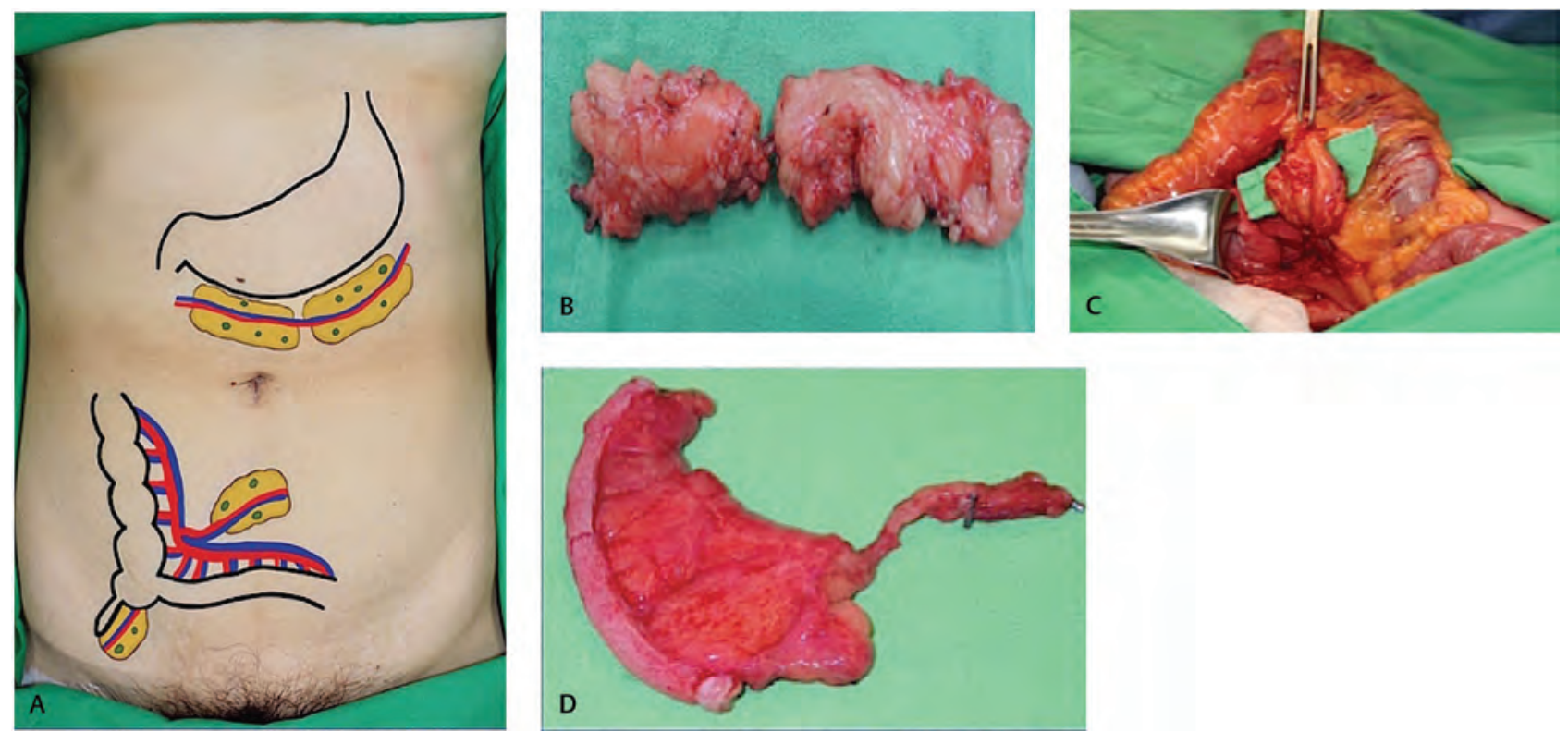

Fig. 4 (A) Anatomical landmarks for intra-abdominal vascularized lymph nodes from the gastroepiploic and ileocecal region. (B)The gastroepiploic lymph node flap was based in the right gastroepiploic vessels. The appendicular and the ileocecal vascularized lymph node flaps were based on the appendicular and ileocolic artery and vein, respectively. (C) For the vascularized appendicular lymph node flap, appendectomy was performed under the operating microscope on a side table. The mesoappendix was dissected and separated from the appendix while paying attention not to devascularize the flap. (D) Vascularized ileocecal lymph node. Both lymph node flaps are alternative options for vascularized lymph node transfer in very selected patients when other more common lymph node flaps are not available. 
early stages of lymphedema. When limb edema is significant, excisional procedures may be necessary to reduce pain and morbidity.

\section{Excisional Procedures}

Excisional procedures have been described for the management of lymphedema since the early 1900. Despite the advancements made and the advent of microsurgical techniques, these procedures remain relevant and can be done to reduce morbidity, risk of infection, and improve quality of life. ${ }^{58}$ Excisional procedures can be done when the physiologic procedures do not offer adequate relief or satisfactory results. Excisional procedures are also done in combination with physiologic procedures to offer better volume reduction. Our first choice of excisional procedure for the LEL is SAL. While patients are advised to use compression garments after SAL, many of our patients don't follow our instructions, mainly due to the warm climate or vocational reasons. In these situations, we have performed radical reduction in lymphedema with preservation of perforators. In selected cases of advanced LEL, the modified Charles' procedure is still a reliable option.

\section{Suction-Assisted Lipectomy}

SAL is the least invasive procedure for lymphedema. It has minimal morbidity and high patient satisfaction when compared with other ablative procedures. Although it provides excellent results, we prefer to perform it in combination with a physiologic procedure. O'Brien et al reported using this technique for the treatment of primary and secondary lymphedema with good results and outcomes. The authors concluded that SAL is a useful and safe procedure that can be done as a primary procedure or in combination with other procedures to achieve good outcomes. ${ }^{59}$ At our institution, a combination of VLNT is followed by laser-assisted liposuction with promising results..$^{60}$ Although there are no standardized guidelines on the optimal timing, we believe that best results are attained when it is performed 1 year after a physiologic procedure as

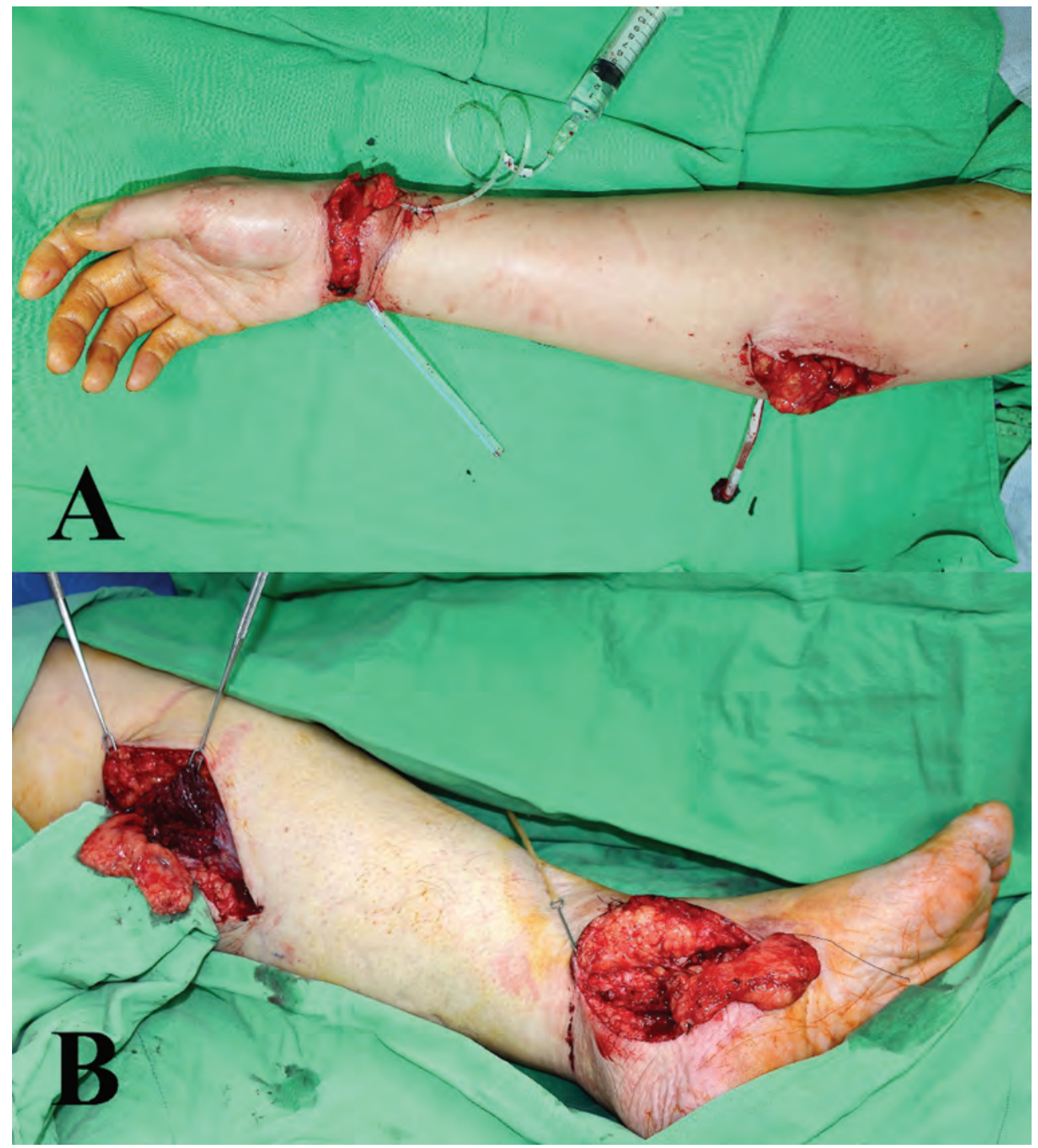

Fig. 5 Double level inset by division in half of a single gastroepiploic vascularized lymph node flap is shown. Upper extremity (A) and lower extremity (B). 

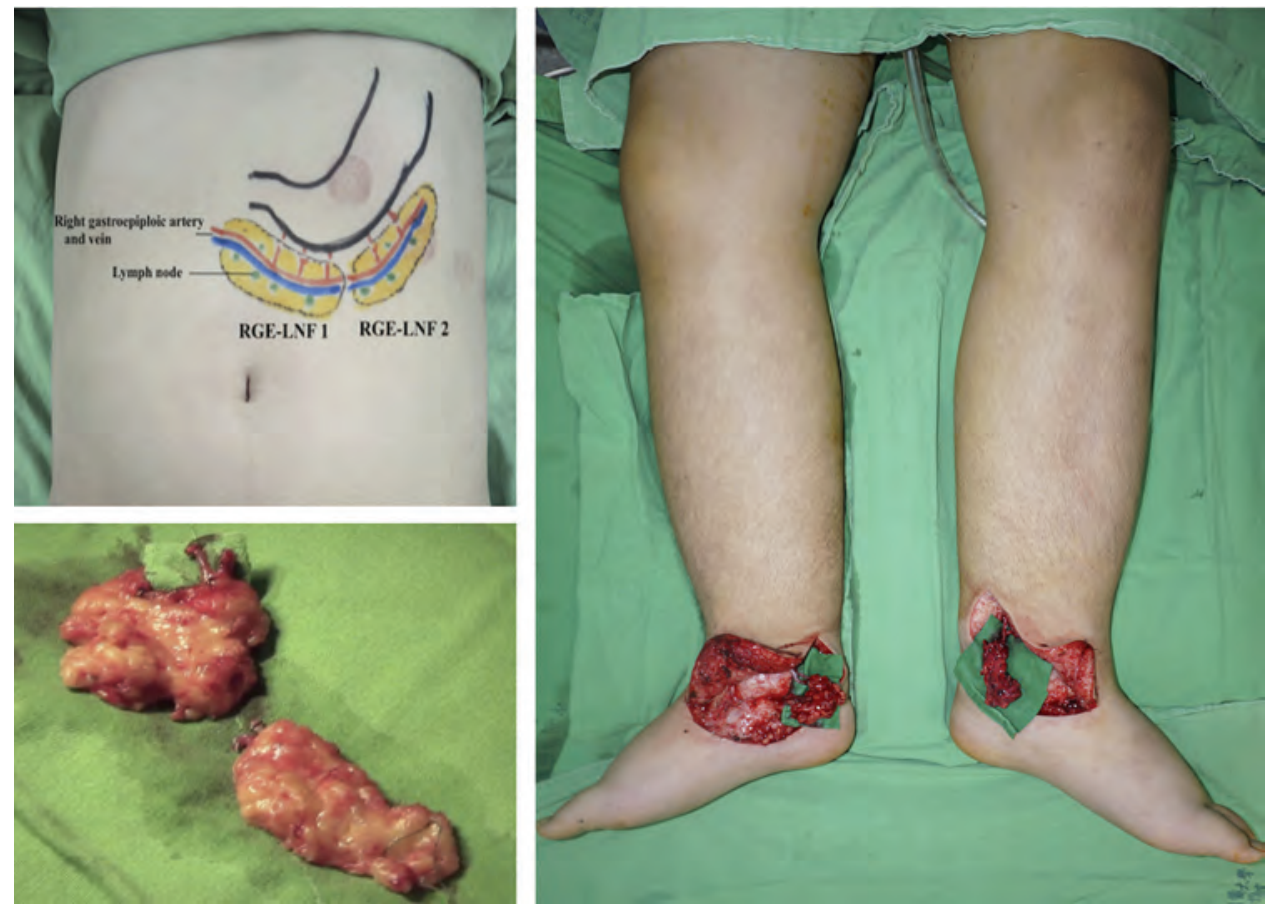

Fig. 6 A double vascularized gastroepiploic lymph node transfer had been performed in one-stage procedure from a single donor site in a patient with bilateral congenital lymphedema.
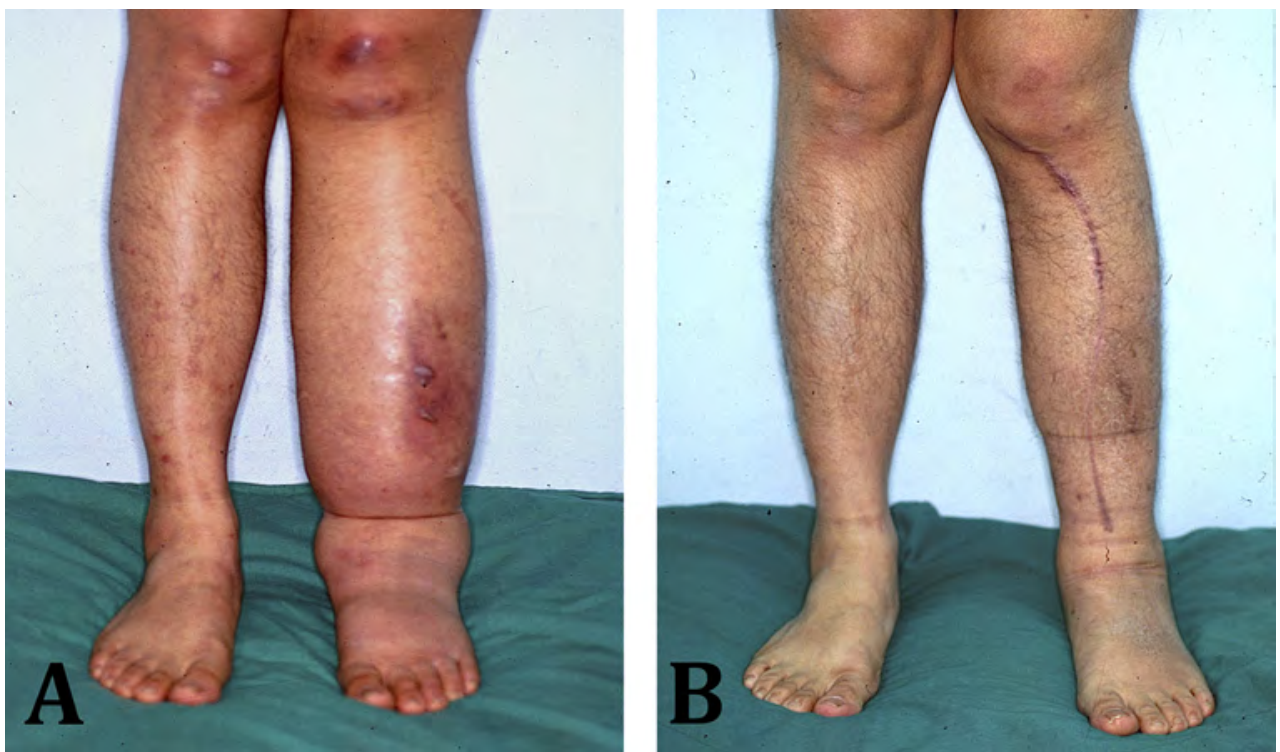

Fig. 7 (A) Preoperative and (B) postoperative appearance following radical reduction in lymphedema with preservation of perforators of the left lower extremity.

this allows the inflammation to subside, volume reduction, and clearance of any infections. The only downside to this procedure is that patients must wear compression garments immediately following the procedure. This makes achieving good results highly dependent on patients' compliance with postoperative recommendations. ${ }^{61,62}$

\section{Radical Reduction in Lymphedema with Preservation of Perforators}

Improved knowledge of the vascular anatomy and perforasome theory ${ }^{63}$ has allowed the introduction of radical reduction of lymphedema procedure with preservation of perforators. This procedure combines both approaches, excisional and microsurgical principles, to manage lymphedema. Several studies have reported excellent and long-lasting outcomes, making this procedure an essential tool in the armamentarium of the reconstructive surgeon. ${ }^{64,65}$ The advantage of this procedure is that it allows for more aggressive debulking of the lymphedematous tissue without damaging essential perforators. The disadvantages, however, lie in the fact that such procedure leaves unsightly scars, prolonged operating time, risk of infection, skin breakdown, and necrosis. This procedure also requires good microsurgical skills ( - Fig. 7). 


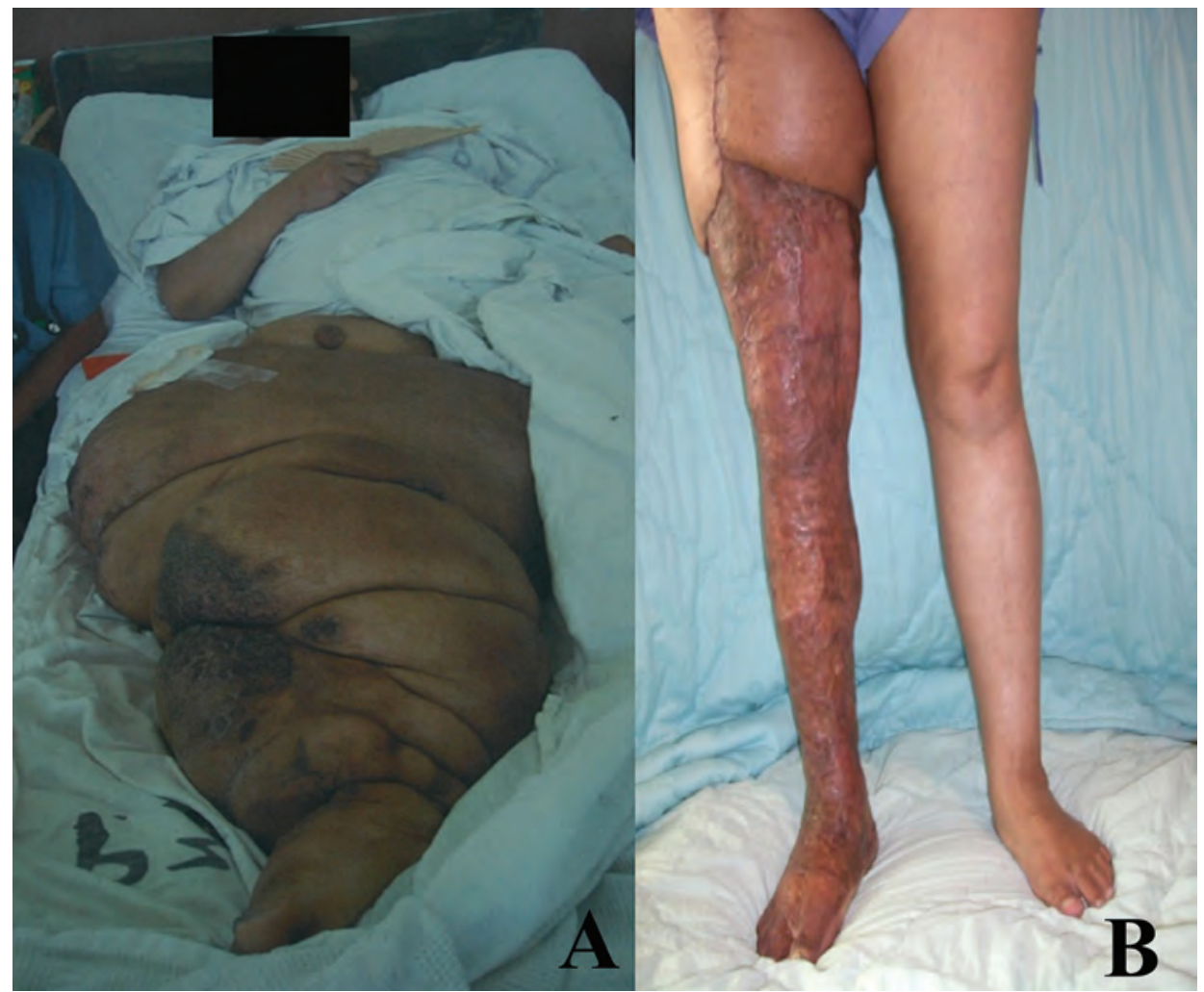

Fig. 8 A 46-year-old woman with chronic right lower limb lymphoedema. (A) Preoperative appearance showing a right lower which was severely affected by lymphedema. The skin was tensed with multiple ulcers and intermittent leakage of lymph. There were deep skin crypts which collected a lot of sebaceous secretion with foul smell. It was very difficult to remove. The bad smell could be sensed when people stand at a distance away. (B) Postoperative picture at 3 years of follow-up after Charles' procedure.

\section{Charles' and Modified Charles' Procedure}

The Charles' procedure is perhaps the oldest and the most radical ablative procedure. It is usually preserved for patients with advanced stage lymphedema and elephantiasis. The main goal is to control and eradicate infection while simultaneously reducing excessive volume. ${ }^{66}$ The Charles' procedure remains a valuable option in carefully selected patients with debilitating lymphedema that is not amenable to treatment using physiological procedures. The modified Charles' procedure, on the other hand, entails the preservation of the greater saphenous vein and its superficial branches. The greater saphenous vein and its branches can be used later as a recipient drain for a transferred lymph node. Patients with severe LEL may benefit from the combination of vascularized lymph node and modified Charles' procedure. ${ }^{67,68}$ Combining the modified Charles' with a vascularized lymph node procedure may prevent some of the side effects of the conventional Charles' procedure such as recurrence, infection, or worsening of the disease. Although excisional procedure could be aggressive at times, they are still valuable options for patients with advanced stages of lymphedema as they improve the quality of life and mobility through drastic reduction in limb volume. ${ }^{67,68}$ (-Figs. 8 and $\mathbf{9}$ ).

\section{Postoperative Management}

Surgical management of lymphedema does not completely eliminate the need for compression therapy. Following physiologic procedures, compression therapy is initiated 1 month postoperatively and immediately following SAL. Patients are usually followed every 3 months postoperatively and the patient is instructed to discontinue compression therapy 2 days prior to their follow-up appointments. If the patient experiences recurrence and excessive lymph accumulation, compressive decongestive therapy is continued for another 3 months. If not, the patient can use compressive therapy during the daytime only for 3 months.

Compression therapy is discontinued if maintenance of limb size for 3 months with daytime therapy was achieved.

\section{Conclusion}

Lymphedema still represents a challenge to the surgeon and the patient alike. It is manageable, however, with a myriad of surgical options that aim to reduce limb volume and restore functionality. Excellent results are within reach when the surgeon and patient are on board with a treatment plan. This 


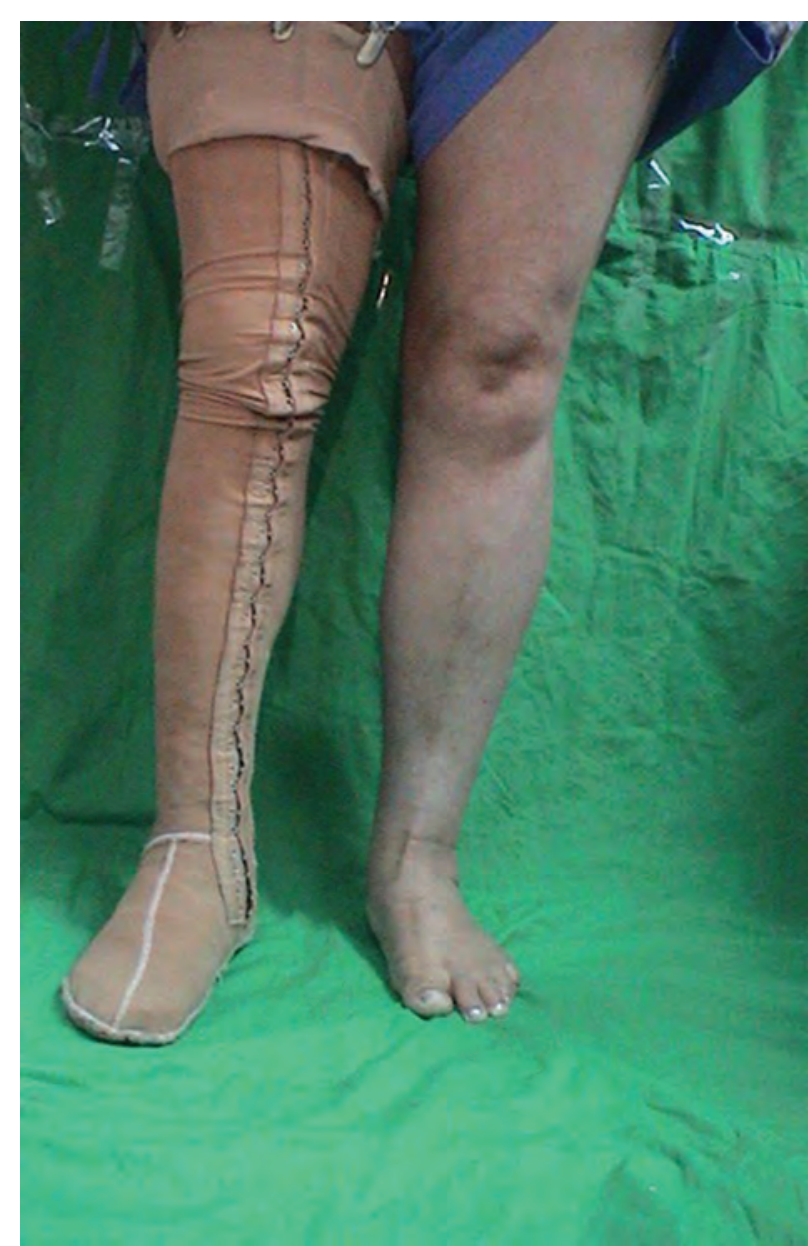

Fig. 9 Compressive stocking to prevent hypertrophic scarring after Charles' procedure.

includes, but not limited to, careful patient selection, choosing the appropriate surgical option, patient compliance with conservative management and appropriate follow-up. Thoughtful individualization and combination of multiple treatment modalities make optimal outcomes attainable in every case.

\section{Conflict of Interest}

None of the authors received any funds or has any financial interests to disclose.

\section{Acknowledgments}

All authors hereby declare not to have any potential conflict of interests and not to have received funding for this work from any of the following organizations: National Institutes of Health $(\mathrm{NIH})$; Wellcome Trust, Howard Hughes Medical Institute (HHMI), and other(s). Each author participated sufficiently in the work to take public responsibility for the content.

\section{References}

1 Shimony A, Tidhar D. Lymphedema: a comprehensive review. Ann Plast Surg 2008;60(2):228

2 International Society of Lymphology. The diagnosis and treatment of peripheral lymphedema: 2013 Consensus Document of the International Society of Lymphology. Lymphology 2013;46(1):1-11
3 Hansen KC, D’Alessandro A, Clement CC, Santambrogio L. Lymph formation, composition and circulation: a proteomics perspective. Int Immunol 2015;27(5):219-227

4 Kung TA, Champaneria MC, Maki JH, Neligan PC. Current concepts in the surgical management of lymphedema. Plast Reconstr Surg 2017;139(4):1003e-1013e

5 Liao S, von der Weid PY. Lymphatic syste an active pathway for immune protection. Semin Cell Dev Biol 2015;38:83-89

6 Blatt J, Powell CM, Burkhart CN, Stavas J, Aylsworth AS. Genetics of hemangiomas, vascular malformations, and primary lymphedema. J Pediatr Hematol Oncol 2014;36(8):587-593

7 Browse NL. The diagnosis and management of primary lymphedema. J Vasc Surg 1986;3(1):181-184

8 Schook CC, Mulliken JB, Fishman SJ, Grant FD, Zurakowski D, Greene AK. Primary lymphedema: clinical features and management in 138 pediatric patients. Plast Reconstr Surg 2011;127(6):2419-2431

9 Wolfe JH, Kinmonth JB. The prognosis of primary lymphedema of the lower limbs. Arch Surg 1981;116(9):1157-1160

10 Babu S, Nutman TB. Immunopathogenesis of lymphatic filarial disease. Semin Immunopathol 2012;34(6):847-861

11 Moffatt CJ, Franks PJ, Doherty DC, et al. Lymphoedema: an underestimated health problem. QJM 2003;96(10):731-738

12 Oremus M, Dayes I, Walker K, Raina P. Systematic review: conservative treatments for secondary lymphedema. BMC Cancer 2012;12:6

13 Warren AG, Brorson H, Borud LJ, Slavin SA. Lymphedema: a comprehensive review. Ann Plast Surg 2007;59(4):464-472

14 Cormier JN, Askew RL, Mungovan KS, Xing Y, Ross MI, Armer JM. Lymphedema beyond breast cancer: a systematic review and meta-analysis of cancer-related secondary lymphedema. Cancer 2010;116(22):5138-5149

15 Biglia N, Zanfagnin V, Daniele A, Robba E, Bounous VE. Lower body lymphedema in patients with gynecologic cancer. Anticancer Res 2017;37(8):4005-4015

16 Abbas S, Seitz M. Systematic review and meta-analysis of the used surgical techniques to reduce leg lymphedema following radical inguinal nodes dissection. Surg Oncol 2011;20(2):88-96

17 Okeke AA, Bates DO, Gillatt DA. Lymphoedema in urological cancer. Eur Urol 2004;45(1):18-25

18 Rasmusson E, Gunnlaugsson A, Blom R, et al. Low rate of lymphedema after extended pelvic lymphadenectomy followed by pelvic irradiation of node-positive prostate cancer. Radiat Oncol 2013;8:271

19 Akita S, Mitsukawa N, Rikihisa N, et al. Early diagnosis and risk factors for lymphedema following lymph node dissection for gynecologic cancer. Plast Reconstr Surg 2013;131(2):283-290

20 Ghanta S, Cuzzone DA, Torrisi JS, et al. Regulation of inflammation and fibrosis by macrophages in lymphedema. Am J Physiol Heart Circ Physiol 2015;308(9):H1065-H1077

21 Tobin MB, Lacey HJ, Meyer L, Mortimer PS. The psychological morbidity of breast cancer-related arm swelling. Psychological morbidity of lymphoedema. Cancer 1993;72(11):3248-3252

22 Pereira de Godoy JM, Braile DM, de Fátima Godoy M, Longo O. Quality of life and peripheral lymphedema. Lymphology 2002;35(2):72-75

23 Fu MR, Ridner SH, Hu SH, Stewart BR, Cormier JN, Armer JM. Psychosocial impact of lymphedema: a systematic review of literature from 2004 to 2011. Psychooncology 2013;22(7):1466-1484

24 Garza R III, Skoracki R, Hock K, Povoski SP. A comprehensive overview on the surgical management of secondary lymphedema of the upper and lower extremities related to prior oncologic therapies. BMC Cancer 2017;17(1):468

25 Farina R III. [Elephantiasis of the legs; treatment by dermofibrolipectomy followed by free skin grafts] An Paul Med Cir 1953;65(4):299-306 
26 Chang DW, Suami H, Skoracki R. A prospective analysis of 100 consecutive lymphovenous bypass cases for treatment of extremity lymphedema. Plast Reconstr Surg 2013; 132(5):1305-1314

27 Suami H, Chang DW. Overview of surgical treatments for breast cancer-related lymphedema. Plast Reconstr Surg 2010;126(6):1853-1863

28 Scaglioni MF, Arvanitakis M, Chen YC, Giovanoli P, Chia-Shen Yang J, Chang EI. Comprehensive review of vascularized lymph node transfers for lymphedema: outcomes and complications. Microsurgery 2018;38(2):222-229

$29 \mathrm{Fu}$ MR. Breast cancer-related lymphedema: symptoms, diagnosis, risk reduction, and management. World J Clin Oncol 2014;5(3):241-247

30 Cormier JN, Xing Y, Zaniletti I, Askew RL, Stewart BR, Armer JM. Minimal limb volume change has a significant impact on breast cancer survivors. Lymphology 2009;42(4):161-175

31 Czerniec SA, Ward LC, Meerkin JD, Kilbreath SL. Assessment of segmental arm soft tissue composition in breast cancer-related lymphedema: a pilot study using dual energy X-ray absorptiometry and bioimpedance spectroscopy. Lymphat Res Biol 2015;13(1):33-39

32 Czerniec SA, Ward LC, Refshauge KM, et al. Assessment of breast cancer-related arm lymphedema--comparison of physical measurement methods and self-report. Cancer Invest 2010;28(1):54-62

33 Maclellan RA, Couto RA, Sullivan JE, Grant FD, Slavin SA, Greene AK. Management of primary and secondary lymphedema: analysis of 225 referrals to a center. Ann Plast Surg 2015;75(2):197-200

34 Chang DW, Masia J, Garza R III, Skoracki R, Neligan PC. Lymphedema: surgical and medical therapy. Plast Reconstr Surg 2016;138(3 Suppl):209S-218S

35 Sundaram PS, Subramanyam P. Lymphoscintigraphy in the evaluation of limb edema. Clin Nucl Med 2013;38(11):891-903

36 Cambria RA, Gloviczki P, Naessens JM, Wahner HW. Noninvasive evaluation of the lymphatic system with lymphoscintigraphy: a prospective, semiquantitative analysis in 386 extremities. J Vasc Surg 1993;18(5):773-782

37 Zaleska MT, Olszewski WL. Indocyanine green near-infrared lymphangiography for evaluation of effectiveness of edema fluid flow under therapeutic compression. J Biophotonics 2018;11(8):e201700150

38 Yamamoto T, Narushima M, Doi K, et al. Characteristic indocyanine green lymphography findings in lower extremity lymphedema: the generation of a novel lymphedema severity staging system using dermal backflow patterns. Plast Reconstr Surg 2011;127(5):1979-1986

39 Campisi C, Bellini C, Campisi C, Accogli S, Bonioli E, Boccardo F. Microsurgery for lymphedema: clinical research and long-term results. Microsurgery 2010;30(4):256-260

40 Koshima I, Inagawa K, Urushibara K, Moriguchi T. Supermicrosurgical lymphaticovenular anastomosis for the treatment of lymphedema in the upper extremities. J Reconstr Microsurg 2000;16(6):437-442

41 Furukawa H, Osawa M, Saito A, et al. Microsurgical lymphaticovenous implantation targeting dermal lymphatic backflow using indocyanine green fluorescence lymphography in the treatment of postmastectomy lymphedema. Plast Reconstr Surg 2011;127(5):1804-1811

42 Chang DW. Lymphaticovenular bypass surgery for lymphedema management in breast cancer patients. Handchir Mikrochir Plast Chir 2012;44(6):343-347

43 Yamamoto T, Koshima I. A prospective analysis of 100 consecutive lymphovenous bypass cases for treatment of extremity lymphedema. Plast Reconstr Surg 2014;133 (6): $887 \mathrm{e}-888 \mathrm{e}$
44 Patel KM, Lin CY, Cheng MH. From theory to evidence: long-term evaluation of the mechanism of action and flap integration of distal vascularized lymph node transfers. J Reconstr Microsurg 2015;31(1):26-30

45 Becker C, Vasile JV, Levine JL, et al. Microlymphatic surgery for the treatment of iatrogenic lymphedema. Clin Plast Surg 2012;39(4):385-398

46 Visuri MT, Honkonen KM, Hartiala P, et al. VEGF-C and VEGF-C156S in the pro-lymphangiogenic growth factor therapy of lymphedema: a large animal study. Angiogenesis 2015;18(3):313-326

47 Liu HL, Pang SY, Lee CC, Wong MM, Chung HP, Chan YW. Orthotopic transfer of vascularized groin lymph node flap in the treatment of breast cancer-related lymphedema: clinical results, lymphoscintigraphy findings, and proposed mechanism. J Plast Reconstr Aesthet Surg 2018;71(7):1033-1040

48 Cheng MH, Huang JJ, Nguyen DH, et al. A novel approach to the treatment of lower extremity lymphedema by transferring a vascularized submental lymph node flap to the ankle. Gynecol Oncol 2012;126(1):93-98

49 Sapountzis S, Singhal D, Rashid A, Ciudad P, Meo D, Chen HC. Lymph node flap based on the right transverse cervical artery as a donor site for lymph node transfer. Ann Plast Surg 2014;73(4):398-401

50 Barreiro GC, Baptista RR, Kasai KE, et al. Lymph fasciocutaneous lateral thoracic artery flap: anatomical study and clinical use. J Reconstr Microsurg 2014;30(6):389-396

51 Ciudad P, Kiranantawat K, Sapountzis S, et al. Right gastroepiploic lymph node flap. Microsurgery 2015;35(6):496-497

52 Ciudad P, Maruccia M, Socas J, et al. The laparoscopic right gastroepiploic lymph node flap transfer for upper and lower limb lymphedema: technique and outcomes. Microsurgery 2017;37(3):197-205

53 Coriddi M, Skoracki R, Eiferman D. Vascularized jejunal mesenteric lymph node transfer for treatment of extremity lymphedema. Microsurgery 2017;37(2):177-178

54 Ciudad P, Manrique OJ, Date S, et al. Vascularized appendicular lymph node transfer for treatment of extremity lymphedema: a case report. Microsurgery 2018;38(5):553-557

55 Ciudad P, Manrique OJ, Agko M, et al. Ileocecal vascularized lymph node transfer for the treatment of extremity lymphedema: a case report. Microsurgery 2019;39(1):81-84

56 Ciudad P, Manrique OJ, Date S, et al. A head-to-head comparison among donor site morbidity after vascularized lymph node transfer: pearls and pitfalls of a 6-year single center experience. J Surg Oncol 2017;115(1):37-42

57 Agko M, Ciudad P, Chen HC. Staged surgical treatment of extremity lymphedema with dual gastroepiploic vascularized lymph node transfers followed by suction-assisted lipectomy-a prospective study. J Surg Oncol 2018;117(6):1148-1156

58 Cormier JN, Rourke L, Crosby M, Chang D, Armer J. The surgical treatment of lymphedema: a systematic review of the contemporary literature (2004-2010) Ann Surg Oncol 2012;19(2):642-651

59 O’Brien BM, Khazanchi RK, Kumar PA, Dvir E, Pederson WC. Liposuction in the treatment of lymphoedema; a preliminary report. Br J Plast Surg 1989;42(5):530-533

60 Nicoli F, Constantinides J, Ciudad P, et al. Free lymph node flap transfer and laser-assisted liposuction: a combined technique for the treatment of moderate upper limb lymphedema. Lasers Med Sci 2015;30(4):1377-1385

61 Brorson H. From lymph to fat: liposuction as a treatment for complete reduction of lymphedema. Int J Low Extrem Wounds 2012;11(1):10-19

62 Boyages J, Kastanias K, Koelmeyer LA, et al. Liposuction for advanced lymphedema: a multidisciplinary approach for complete reduction of arm and leg swelling. Ann Surg Oncol 2015;22(Suppl 3):S1263-S1270 
63 Saint-Cyr M, Wong C, Schaverien M, Mojallal A, Rohrich RJ. The perforasome theory: vascular anatomy and clinical implications. Plast Reconstr Surg 2009;124(5):1529-1544

64 Salgado CJ, Sassu P, Gharb BB, Spanio di Spilimbergo S, Mardini S, Chen HC. Radical reduction of upper extremity lymphedema with preservation of perforators. Ann Plast Surg 2009;63(3):302-306

65 Salgado CJ, Mardini S, Spanio S, Tang WR, Sassu P, Chen HC. Radical reduction of lymphedema with preservation of perforators. Ann Plast Surg 2007;59(2):173-179

66 Kobayashi MR, Miller TA. Lymphedema. Clin Plast Surg 1987;14(2):303-313
67 Sapountzis S, Ciudad P, Lim SY, et al. Modified Charles procedure and lymph node flap transfer for advanced lower extremity lymphedema. Microsurgery 2014;34(6):439-447

68 Yeo MS, Lim SY, Kiranantawat K, Ciudad P, Chen HC. A comparison of vascularized cervical lymph node transfer with and without modified Charles' procedure for the treatment of lower limb lymphedema. Plast Reconstr Surg 2014;134(1):171e-172e 